\title{
The yeast multidrug resistance pump, Pdr5p, confers reduced drug resistance in erg mutants of Saccharomyces cerevisiae
}

\author{
Rupinder Kaur and Anand K. Bachhawat \\ Author for correspondence: Anand K. Bachhawat. Tel: +91 172 690004/690908. \\ Fax: +91 172 690585/690632. e-mail: abachhawat@excite.com
}

Institute of Microbial Technology, Sector 39-A, Chandigarh - 160036, India

\begin{abstract}
Mutants of Saccharomyces cerevisiae bearing lesions in the ergosterol biosynthetic pathway exhibit a pleiotropic drug-sensitive phenotype. This has been reported to result from an increased permeability of the membranes of the mutant strains to different drugs. As disruption of the yeast multidrug resistance protein, Pdr5p, results in a similar pleiotropic drug-sensitive phenotype, the possibility that Pdr5p may be functioning with a reduced efficiency in these altered sterol backgrounds was examined. To do this, the function of Pdr5p in isogenic strains of $S$. cerevisiae that have disruptions in the late stages of the ergosterol biosynthesis pathway (ERG6, ERG2, ERG3, ERG4) was studied. A reduced ability of Pdr5p to confer resistance to different drugs in these strains was observed, which did not appear to be dependent solely on the permeability of the membrane towards the drug. A simultaneous examination was made of how the lipid composition might be altering the efficiency of Pdr5p by similar studies in strains lacking phosphatidylserine synthase (encoded by $\mathrm{CHO1}$ ). The results indicated that the drug sensitivity of the erg strains is, to a significant extent, a result of the reduced efficiency of the Pdr5p efflux pump, and that the membrane environment plays an important role in determining the drug resistance conferred by Pdr5p.
\end{abstract}

Keywords: Saccharomyces cerevisiae, multidrug resistance pump, membrane environment, sterols, erg mutants

\section{INTRODUCTION}

Multidrug resistance (MDR), the acquisition of simultaneous resistance to a wide variety of structurally and functionally unrelated compounds, is often associated with the overexpression of one or more members of a superfamily of ATP-binding casssette transporters (Gottesman \& Pastan, 1993). These proteins are comprised of 12 membrane-spanning $\alpha$-helices and two ATP-binding domains (Borst \& Schinkel, 1997). MDR is a ubiquitous phenomenon and the membrane proteins responsible for this phenomenon are conserved throughout the evolutionary scale. The mechanism by which these proteins are able to confer resistance to such a structurally diverse range of compounds is still not known (Gottesman et al., 1996). Structure-function

Abbreviations: DPH, diphenylhexatriene; TMA-DPH, trimethylammonium diphenylhexatriene; MDR, multidrug resistance; P-gp, phosphoglycoprotein; wt, wild-type. analyses from different groups (Loo \& Clarke, 1993; Beaudet \& Gios, 1995; Hanna et al., 1996; Kwan \& Gros, 1998) have not identified any single domain that is responsible for the diverse substrate specificity of MDR pumps, although mutations affecting substrate specificity appear to be clustered around the transmembrane domains and the substrates have been shown to bind within the membrane bilayer (Sharom, 1997).

The yeast Saccharomyces cerevisiae has also been shown to contain a MDR efflux pump, Pdr5p, that belongs to the family of $\mathrm{ABC}$ transporters. Pdr5p has been cloned independently by several groups (Bissinger \& Kuchler, 1994; Balzi et al., 1994; Hirata et al., 1994; Kralli et al., 1995) and has been shown to confer resistance to a wide range of compounds and metal ions (Mahe et al., 1996). Overproduction of this protein confers pleiotropic drug resistance, whilst disruption of the gene encoding it leads to a pleiotropic drug-sensitive phenotype. Recent work with Pdr5p, however, could not identify any single domain that affects the substrate specificity of this pump 
and it was suggested that the folded structure of the Pdr $5 p$ protein, and possibly the membrane composition, might play an important role in determining its substrate specificity (Egner et al., 1998). Yeast erg mutants, which carry mutations in the ergosterol biosynthetic pathway, are resistant to antifungal antibiotics that target this pathway, but are otherwise pleiotropically drug-sensitive to a wide variety of structurally diverse compounds. This has been reported to be due to an increased permeability of their membranes to different drugs (Bard et al., 1978). However, considering that disruption of the yeast $\mathrm{Pdr} 5$ pump also results in similar pleiotropic drug sensitvities, and the possible importance of the membrane environment in the functioning of these pumps, we decided to re-examine the drug-sensitive phenotypes of the erg mutants and investigate how the Pdr5 pump functions in these strains. An earlier investigation had reported that ergosterol, the major sterol of the yeast plasma membrane, inhibits the drug-binding ability of mammalian phosphoglycoproteins (P-gps) when expressed in yeast (Saeki et al., 1991); however, this has not been substantiated by later workers (Kuchler \& Thorner, 1992). A possible role of lipids in the modulation of the drug-binding activity of P-gps has been suggested by the observations that many characteristics of P-gps, including the pattern of ATPase stimulation or inhibition by drugs, is affected by the lipid environment (Doige et al., 1993; Saeki et al., 1992; Urbatsch \& Senior, 1995).

Our results, described in this report, indicate that the membrane environment/composition plays a significant role in determining the functioning of MDR pumps, and that the drug sensitivity of erg mutants is, to a large extent, due to the decreased drug efflux mediated by $\mathrm{Pdr} 5 \mathrm{p}$ in these strains.

\section{METHODS}

Chemicals. The chemicals used were of analytical grade. All media components were either purchased from HiMedia or Difco. Rhodamine 6G, cycloheximide, crystal violet, emetine and $\beta$-oestradiol were obtained from Sigma. Diphenylhexatriene (DPH) and trimethylammonium diphenylhexatriene (TMA-DPH) were obtained from Molecular Probes. Oligonucleotides were purchased from Ransom Hill Biosciences. Restriction/modification enzymes were obtained from New England Biolabs.

Yeast strains, media and growth conditions. The yeast strains used in this study are listed in Table 1 . They were grown at $30^{\circ} \mathrm{C}$ and maintained on yeast extract/peptone/dextrose medium (YPD). YPD and synthetic media (SD) were prepared as described by Rose et al. (1990).

Stock solutions of crystal violet, cycloheximide and emetine were prepared in water, whilst rhodamine $6 \mathrm{G}$ and $\beta$-oestradiol solutions were made in ethanol. Plates containing different compounds were made by adding the required volume of the stock solution of the compound to YPD medium during pouring.

Recombinant DNA methods. DNA manipulations including PCR were essentially carried out as described by Sambrook et al. (1989). Yeast genomic DNA was isolated by the glass bead lysis method (Rose et al., 1990). Yeast transformations were carried out by the lithium acetate method (Ito et al., 1983).

\section{Strain construction}

(1) Construction of erg $4 \Delta$ strain. Plasmid $\mathrm{pCM} 2$ containing the ERG4 gene disrupted with URA3 with flanking repeats (obtained from C. Marcireau, Rhone-Poulenc Rorer, VitrySur-Seine, France) was cleaved with $\mathrm{Sacl} / \mathrm{HpaI}$ and the $6.94 \mathrm{~kb}$ erg 4A::URA3 fragment was transformed into strain $\mathrm{ABC} 709$ and selected for uracil prototrophy. The disruption at the ERG4 locus was confirmed by phenotypic analysis (drug sensitivity) and the $U R A 3$ region was popped out by selecting on 5-fluoroorotic acid plates, rendering erg $4 \Delta$ a uracil auxotroph. The disruptant strain thus obtained was crossed with strain $\mathrm{ABC} 710$ and tetrads were dissected to obtain an $\operatorname{erg} 4 \Delta$ $\mathrm{ABC} 283$ strain in a protease-deficient background.

(2) Construction of erg3s strain. The fragment containing erg3 $3:: L E U 2$ was amplified from strain ABC 102 (erg3 $3:: L E U 2$, obtained from L. Parks, North Carolina State University, Raleigh, USA) with the primers for the flanking regions of this fragment. Strain ABC 287 [constructed from a cross of $A B C 229$ and $A B C 710$, which was used as a wild-type (wt) strain] was transformed with the purified $2.4 \mathrm{~kb}$ PCR product and selected for leucine prototrophy. The disruptant $\mathrm{ABC} 261$ was confirmed by phenotypic characterization and PCR.

(3) Construction of erg2 $2 \Delta$ strain. The disruption of $\operatorname{erg} 2 \Delta:: L E U 2$ was done by introducing a $2.2 \mathrm{~kb} L E U 2$ gene at the $N d e I$ site within the coding region of $E R G 2$ in the plasmid pFLA 2-7 (the plasmid was obtained from J. Heinisch, Heinrich Heine Universität, Dusseldorf, Germany). A $3.2 \mathrm{~kb}$ StuI-PstI fragment containing the $\operatorname{erg} 2 \Delta:: L E U 2$ construct was used to transform strain $\mathrm{ABC} 709$ to leucine prototrophy. The strain was confirmed for the disruption at the right locus by phenotypic analysis and PCR. The disruptant was mated with strain $\mathrm{ABC} 710$ and tetrad dissection was done to obtain an erg2 $\triangle$ strain (ABC 271) in a protease-deficient background.

(4) Construction of erg6 strain. An ERG6 (SED6) plasmid was obtained from H. Pelham, MRC, Cambridge, UK, and an $E R G 6$ disruption was made by inserting a $L E U 2$ fragment into the $K p n I$ site of $E R G 6$ that was first subcloned into the vector pGEX-2T. A $3.2 \mathrm{~kb}$ ScaI-BamHI fragment containing the erg6 $6:: L E U 2$ fragment was transformed into $\mathrm{ABC} 709$ and selected for leucine prototrophy. The disruption at the ERG6 locus was confirmed by PCR. An erg6 $\Delta$ strain (ABC 265) in a protease-deficient background was obtained by crossing the resultant disruptant with $\mathrm{ABC} 710$ and subsequently dissecting tetrads.

(5) Construction of $p d r 5 \Delta$ strain. A diploid was made by crossing $\mathrm{ABC} 710$ with $\mathrm{ABC} 152$ (pdr5s::TRP1) and a $3.2 \mathrm{~kb}$ Scal$B a m \mathrm{HI}$ fragment of the plasmid bearing erg6 $6:: L E U 2$ was transformed into the diploid strain. The transformants were selected for leucine prototrophy and then plated on the sporulation medium. Tetrad dissection was done to obtain a $p d r 5 \Delta$ (ABC 288) strain in a protease-deficient background.

(6) Construction of cho1 1 strain. The cho1 $1:$ :LEU2 strain was constructed by the PCR-mediated direct gene disruption method (Baudin et al., 1993). The primers CHDEL1 ( $5^{\prime}$ CACAGAGACGAAAATGACGGGTATGCCTCAGATGAAGTTCGACTACGTCGTAAGGCCG 3') and CHDEL2 (5' GACACAATATGCCATACCCAACACCAAAGCTAGAGTGGTAATGGAATCCCAACAATTACA 3') were used 
Table 1. Yeast strains used in the present study

\begin{tabular}{|c|c|c|}
\hline Strain & Genotype & Source* \\
\hline $\begin{array}{l}\mathrm{ABC} 102 \\
(\mathrm{SY} 12-\mathrm{D})\end{array}$ & 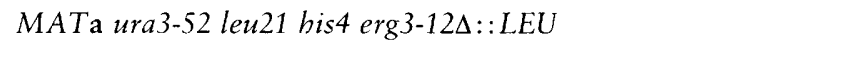 & L. Parks \\
\hline $\begin{array}{l}\mathrm{ABC} 152 \\
(\text { YKKA-7) }\end{array}$ & 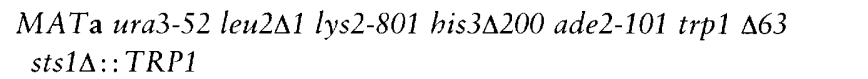 & K. Kuchler \\
\hline $\begin{array}{l}\mathrm{ABC} 154 \\
(\mathrm{YPH} 499)\end{array}$ & MATa ura3-52 leu $2 \Delta 1$ lys2-801 his $3 \Delta 200$ ade2-101 trp1 $\Delta 63$ & K. Kuchler \\
\hline $\mathrm{ABC} 229$ & MATа ura3-52 leu $2 \Delta 1$ lys2-801 his3 $\Delta 200$ erg $2 \Delta:: L E U 2$ & This study \\
\hline $\mathrm{ABC} 261$ & $\begin{array}{l}\text { MATa ura3-52 leu } 2 \Delta 1 \text { lys2-801 bis } 3 \Delta 200 \text { pep } 4:: H I S 3 \text { prb1 } 1.6 R \\
\text { can1 erg } 3 \Delta: \text { :LEU2 }\end{array}$ & This study \\
\hline $\mathrm{ABC} 265$ & 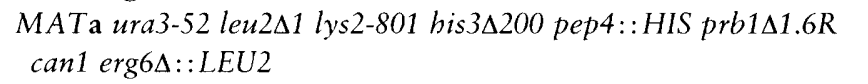 & This study \\
\hline $\mathrm{ABC} 271$ & $\begin{array}{l}\text { MATa ura3-52 leu } 2 \Delta 1 \text { lys2-801 his } 3 \Delta 200 \text { pep } 4: \text { : HIS prb1 } 1.6 \mathrm{R} \\
\text { can1 erg } 2 \Delta:: \text { LEU2 }\end{array}$ & This study \\
\hline $\mathrm{ABC} 283$ & $\begin{array}{l}\text { MATa ura3-52 leu } 2 \Delta 1 \text { lys2-801 bis } 3 \Delta 200 \text { pep } 4:: \text { HIS } 3 \text { prb1 } 1.6 R \\
\text { can1 erg } 4 \Delta\end{array}$ & This study \\
\hline $\mathrm{ABC} 287$ & 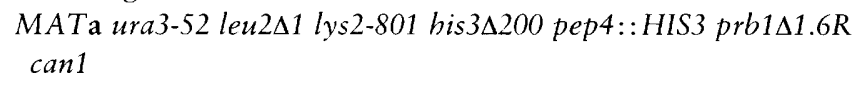 & This study \\
\hline $\mathrm{ABC} 288$ & 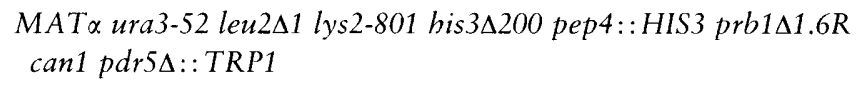 & This study \\
\hline $\mathrm{ABC} 625$ & $\begin{array}{l}\text { MATa ura3-52 leu } 2 \Delta 1 \text { lys } 2-801 \text { bis } 3 \Delta 200 \text { ade } 2-101 \text { trp } 1 \Delta 63 \\
\text { cho1 } \Delta: \text { :TRP1 }\end{array}$ & This study \\
\hline $\mathrm{ABC} 626$ & 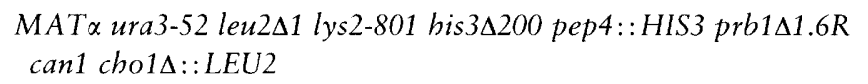 & This study \\
\hline $\begin{array}{l}\mathrm{ABC} 706 \\
(\mathrm{BRS} / 188)\end{array}$ & 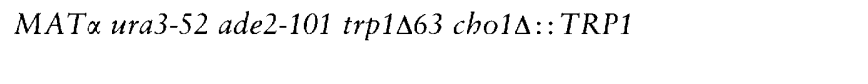 & S. Henry \\
\hline $\begin{array}{r}\mathrm{ABC} 709 \\
(\mathrm{BJ} 5418)\end{array}$ & MATa ura3-52 leu2 21 lys 2-801 his $3 \Delta 200$ & E. Jones \\
\hline $\begin{array}{l}\mathrm{ABC} 710 \\
(\mathrm{BJ} 5458)\end{array}$ & 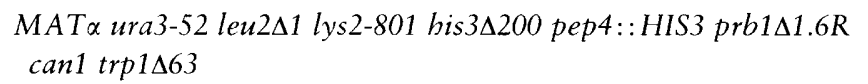 & E. Jones \\
\hline
\end{tabular}

* L. Parks, North Carolina State University, Raleigh, USA; K. Kuchler, University and Biocenter of Vienna, Austria; S. Henry and E. Jones, Carnegie Mellon University, Pittsburgh, USA.

for making the disruption. PCR was done from a LEU2-based plasmid, pSP1, and the $2 \cdot 2 \mathrm{~kb}$ amplified product was used to transform $\mathrm{ABC} 287$ strain to leucine prototrophy. The putative disruptant (leucine prototroph) (ABC 626) was checked for disruption by phenotypic analysis (choline auxotrophy) and PCR.

To construct strain cho1 $1:: T R P 1$ in a background that was not protease-deficient, PCR was done on strain ABC 706 (cho1 1 : :TRP1) with CHFOR (5' TCACATGGACCCATCTAAAGA 3') and CHREV (5' TTTGACGCCAGGCATGAACAA $\left.3^{\prime}\right)$ primers. The amplified product $(2.6 \mathrm{~kb})$ having cho1 $1:$ TRP1 was used to transform strain ABC 154 to tryptophan prototrophy. The disruption at the right locus was confirmed by phenotypic characterization and PCR in strain $\mathrm{ABC} 625$.

Construction of plasmid. The plasmid PDR5/YEplac195 was constructed by digesting pSTSI (obtained from K. Kuchler, University and Biocenter of Vienna, Austria) with $S p h \mathrm{I} / A p a \mathrm{I}$, purifying the $7.5 \mathrm{~kb}$ fragment and cloning in the $S m a \mathrm{I}$ site of the multicopy plasmid YEplac195 (Gietz \& Sugino, 1988).

In vivo drug sensitivity assay. Pdr $5 \mathrm{p}$-mediated resistance to different compounds was tested by spotting serial dilutions of yeast culture onto plates containing drugs. The transformants were pregrown in SD broth lacking uracil to late-exponential phase and then reinoculated into fresh medium to a cell concentration of $5 \times 10^{6}$ cells $\mathrm{ml}^{-1}$. After incubation for $6-7 \mathrm{~h}$ at $30^{\circ} \mathrm{C}$, the optical density was measured at $600 \mathrm{~nm}$ and the number of cells per $\mathrm{ml}$ of culture was calculated. Serial dilutions containing $10^{7}, 10^{6}, 10^{5}$ and $10^{4}$ cells $\mathrm{ml}^{-1}$ were then made in sterile water and $10 \mu \mathrm{l}$ of each serially diluted culture was spotted onto YPD plates containing either the solvent or the drug, and onto SD lacking uracil (SD - ura) selection plates to check for plasmid stability.

MIC estimations. The MIC of a drug was defined as the minimum concentration at which no growth was observed when $10 \mu \mathrm{l}$ of the second dilution (i.e. $10^{6}$ cells $\mathrm{ml}^{-1}$ ) of a culture was spotted onto the plate containing the drug.

Rhodamine 6 G efflux assay in whole cells. The rhodamine efflux study was essentially carried out as described by Kolaczkowski et al. (1996) with a few modifications. The strains were pregrown in YPD broth for 12-14 h and then reinoculated into fresh media to an initial $\mathrm{OD}_{600}$ of $0 \cdot 2$. After incubation for $5-6 \mathrm{~h}$ at $30^{\circ} \mathrm{C}$, equal numbers of cells from an exponential-phase culture were harvested, washed four times with water and resuspended (wet wt $=40 \mathrm{mg}$ ) in $0.5 \mathrm{ml}$ 
HEPES buffer (50 mM HEPES-NaOH, pH 7-0). 2-Deoxy-Dglucose and rhodamine $6 \mathrm{G}$ were added to a final concentration of $5 \mathrm{mM}$ and $10 \mu \mathrm{M}$, respectively, and incubated at $25^{\circ} \mathrm{C}$ for $2 \mathrm{~h}$. After incubation, rhodamine-6G-loaded cells were quickly spun down, washed twice with HEPES buffer and finally resuspended in $4 \mathrm{ml}$ HEPES buffer. The final suspension was aliquoted into two parts ( $2 \mathrm{ml}$ each), $1 \mathrm{mM}$ glucose was added to one aliquot to initate the active, Pdr5p-mediated efflux of rhodamine $6 \mathrm{G}$. The cell suspension was incubated at room temperature. At time zero, i.e. immediately after the addition of glucose, a $0.5 \mathrm{ml}$ sample was withdrawn from both aliquots (with and without glucose), cells were quickly spun down and the fluorescence of rhodamine extruded in the assay buffer was measured at an excitation and emission wavelength of $529 \mathrm{~nm}$ and $553 \mathrm{~nm}$, respectively (slit width $=5$ ) on a Perkin-Elmer LS $50 \mathrm{~B}$ spectrofluorimeter. After 7 and $21 \mathrm{~min}$ further incubation, a $0.5 \mathrm{ml}$ sample was again withdrawn, cells were removed by centrifugation and the fluorescence of rhodamine $6 \mathrm{G}$ extruded in the supernatant was measured.

Plasma membrane fluidity measurement. The fluidity of the yeast plasma membrane was measured in the whole cells using DPH and TMA-DPH as the fluorescent probes (Obrenovitch et al., 1978; Kuhry et al., 1985). The solutions of the probes were prepared in DMSO. Yeast cultures were grown overnight in YPD at $30^{\circ} \mathrm{C}$ and then reinoculated into YPD at an $\mathrm{OD}_{600}$ of approximately $0 \cdot 2-0 \cdot 3$. After incubation for $5-6 \mathrm{~h}$, cells were harvested, washed twice with sterile water and then suspended in buffer 1 (100 mM sodium phosphate, $100 \mathrm{mM}$ sodium chloride, $1 \mathrm{mM}$ EDTA, $\mathrm{pH} \mathrm{7.4)}$ to a density of $8 \mathrm{mg}$ wet weight $\mathrm{ml}^{-1}$. The cells were incubated at $20^{\circ} \mathrm{C}$ for $5 \mathrm{~min}$ and the DPH and TMA-DPH probes were added to a final concentration of $2 \mu \mathrm{M}$ or $5 \mu \mathrm{M}$, respectively. The same volume of the solvent (DMSO) was added to the cells as a control. Incubation was continued for $20 \mathrm{~min}$ at $20^{\circ} \mathrm{C}$, after which the cells were immediately washed twice with buffer 1 and then suspended in the same volume of buffer 1. Fluorescence polarization was measured on a Perkin-Elmer LS 50 B spectrofluorimeter. The excitation and emission wavelengths were $360 \mathrm{~nm}$ and $450 \mathrm{~nm}$, respectively. The measured fluorescence intensities were corrected for background fluorescence and light scattering from the unlabelled sample, i.e. the sample treated with the solvent alone. The degree of steady-state anisotropy $\left(r_{s}\right)$ was calculated as described by Haggerty et al. (1978)

\section{RESULTS}

\section{Effect of Pdr5p overexpression on drug resistance in erg strains}

Strains with disruptions in the late steps of the ergosterol biosynthetic pathway are viable and accumulate sterols other than ergosterol in the membrane (Lees et al., 1995). The predominant sterols that accummulate are the ones shown in Fig. 1. These strains have also been demonstrated to display an increased sensitivity to many drugs owing to an increased permeability of the membranes (Bard et al., 1978). We decided to examine if the yeast MDR protein, Pdr5p, might be affected in the membranes with these different sterol contents. We constructed disruptions in the ERG6, ERG2, ERG3 and ERG4 genes as described in Methods. The strains were constructed in a vacuolar-protease-deficient ( $p e p 4$ prb1) background to minimize differences in the turnover rates of Pdr5p; the turnover of Pdr5p has been shown to

\author{
Zymosterol \\ $\downarrow$ ERG6 \\ Fecosterol \\ $\downarrow E R G 2$ \\ Episterol \\ $\downarrow E R G 3$ \\ Ergosta-5,7,24(28)-trienol \\ $\downarrow$ ERG5 \\ Ergosta-5,7,22,24(28)-tetraenol \\ $\downarrow E R G 4$ \\ Ergosterol
}

Fig. 1. Schematic representation of the late stages of the ergosterol biosynthesis pathway from zymosterol to ergosterol. ERG6, ERG2,ERG3, ERG5 and ERG4 encode S-adenosyl methionine methyltransferase, $\mathrm{C}-8$ sterol isomerase, sterol $\Delta 5$ desaturase, C-22 sterol desaturase and sterol-24(28) reductase, respectively.

occur in the vacuole (Egner et al., 1995; Egner \& Kuchler, 1996).

Pdr5p was overexpressed in different erg strains by introducing the PDR5 gene on a URA3-based multicopy plasmid, transforming the different erg strains with this plasmid, and then examining the resistance conferred to different drugs. We used drug resistance conferred by $\operatorname{Pdr} 5 \mathrm{p}$ overexpression in these strains as a means of evaluating Pdr5p function. Overexpression of Pdr5p itself did not affect the growth of the strains relative to the vector only, nor were there any differences in plasmid stability in the different strains (data not shown).

We observed that all the erg strains showed an increased sensitivity to cycloheximide although to different extents (Fig. 2). Control experiments without drugs, or with only the solvents in which the drugs were dissolved, were carried out in these and subsequent experiments, to eliminate any differences that might have occurred in the absence of drugs (data not shown). As additional controls, the resistance conferred in wt and $p d r 5 \Delta$ strains was also simultaneously determined and these data are included in all the figures. The $\operatorname{erg} 6 \Delta$ and $\operatorname{erg} 4 \Delta$ strains were the most sensitive, and the sensitivity of the $p d r 5 \Delta$ strain was also comparable to that of the erg6 $\Delta$ and $\operatorname{erg} 4 \Delta$ strains. The sensitivities of the strains are a reflection of the intracellular accumulation of the drug under steady-state conditions. When we overexpressed $\operatorname{Pdr} 5 \mathrm{p}$ in these strains, we found that overexpression of the Pdr $5 \mathrm{p}$ in these different strains did not lead to drug resistance to the same levels (Fig. 2). Pdr5p overexpression led to significantly higher levels of drug resistance in the $\operatorname{erg} 4 \Delta$ strain as compared to the erg6 $\Delta$ strain, and even as compared to the erg $2 \Delta$ strain, which otherwise displayed lesser sensitivity. In the $p d r 5 \Delta$ 


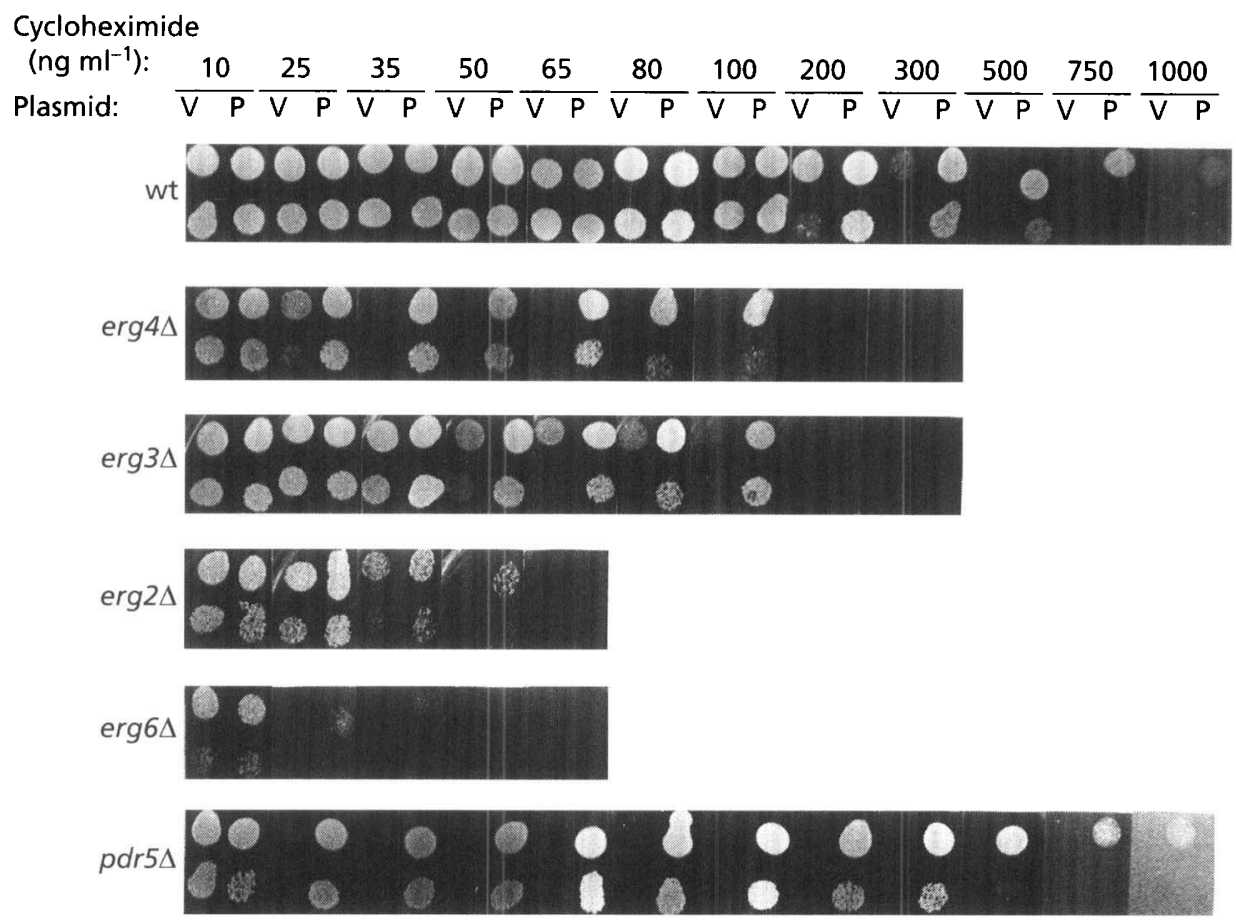

Fig. 2. Pdr5p-mediated cycloheximide resistance in wt, erg $4 \Delta$, erg $3 \Delta$, erg $2 \Delta$, erg $6 \Delta$ and pdr5 strains of $S$. cerevisiae. Strains transformed either with the control plasmid YEplac195 (V) or with the PDR5-overexpressing plasmid PDR5/YEplac195 (P) were grown to exponential phase in SD-ura at $30^{\circ} \mathrm{C}$. An equal number of cells of each strain was harvested, resuspended in sterile water to a density of $1 \times 10^{7}$ cells $\mathrm{ml}^{-1}$ and $10 \mu \mathrm{l}$ of undiluted cell suspension, $1: 10$, 1:100 and 1:1000 dilutions was spotted onto YPD plates containing different concentrations of cycloheximide. Growth

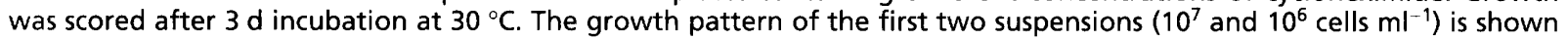
in the first and second rows, respectively.

strain, however, which otherwise has no altered sterols in the membrane, the level of resistance conferred was up to the wt levels. These results indicate firstly that alteration in the sterol content of the cells led to a decreased drug resistance conferred by Pdr5p as compared to the cells having ergosterol in their membranes, and secondly that the decreased resistance was not a simple consequence of increased permeability and sensitivity of the strains to the drug.

To examine whether our observation with cycloheximide was a general phenomenon, we extended this study to other structurally unrelated drugs, crystal violet, emetine and oestradiol. The conclusions we reached with crystal violet (data not shown), were very similar to those with cycloheximide. In the case of emetine, however, $\mathrm{Pdr} 5 \mathrm{p}$ virtually failed to confer any significant resistance in $\operatorname{erg} 6 \Delta$ and $\operatorname{erg} 2 \Delta$ strain backgrounds (Fig. 3), although it could mediate efflux of the drug in the other strain backgrounds. The results with oestradiol were interesting in that we were able to observe oestradiol toxicity for the first time in yeast using the erg6s strain. However, we observed that oestradiol toxicity could be effectively reversed even in an $\operatorname{erg} 6 \Delta$ background by Pdr5p overexpression (Fig. 4), indicating that Pdr5p was functioning in this background to efflux some, but not all of the drugs.

\section{Rhodamine 6G efflux in ergosterol-deficient strains of $S$. cerevisiae}

Since the decreased drug resistance conferred by Pdr $5 p$ was indirect evidence of Pdr5p efficiency under steadystate conditions, we decided to measure Pdr $5 p$ efficiency directly by monitoring the $\mathrm{Pdr} 5 \mathrm{p}$-mediated efflux of rhodamine $6 \mathrm{G}$ in erg backgrounds at different time intervals.

Rhodamine 6G is a known substrate of $\mathrm{Pdr} 5 \mathrm{p}$ and is reported to be effluxed by Pdr5p in an energy-dependent manner (Kolaczkowski et al., 1996). To check the functioning of Pdr5p in altered sterol backgrounds, we carried out the rhodamine efflux assay in erg strains along with the wt and $p d r 5 \Delta$ strains serving as the positive and negative controls. The energy-dependent Pdr $5 p$-driven extrusion of rhodamine was initiated by the addition of $1 \mathrm{mM}$ glucose and fluorescence of extruded dye in the assay buffer was measured after different time intervals. The $\operatorname{erg} 4 \Delta, \operatorname{erg} 2 \Delta$ and $\operatorname{erg} 6 \Delta$ strains were found to efflux rhodamine $6 \mathrm{G}$ at a slower rate than the wt cells while erg3s cells showed a relatively higher rate of efflux as compared to other erg strains (Table 2). Rhodamine efflux was seen in the wt, $\operatorname{erg} 4 \Delta$ and $\operatorname{erg} 3 \Delta$ strains $7 \mathrm{~min}$ after glucose addition; however, very little efflux was seen in $\operatorname{erg} 2 \Delta$ and $\operatorname{erg} 6 \Delta$ strains after $7 \mathrm{~min}$. We also examined the efflux after 
Emetine

$\begin{array}{lllllllll}\left(\mu \mathrm{g} \mathrm{m}^{-1}\right): & 50 & 100 & 200 & 300 & 500 & 750 & 1000 & 2000\end{array}$

Plasmid:

wt

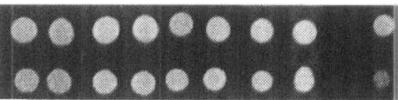

$\operatorname{erg} 4 \Delta$

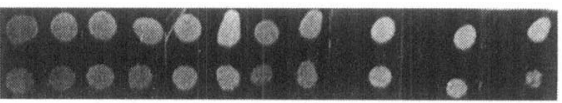

$\operatorname{erg} 3 \Delta$

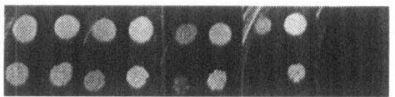

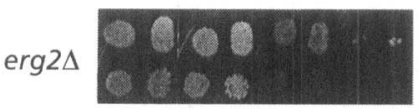

$\operatorname{erg} 6 \Delta$

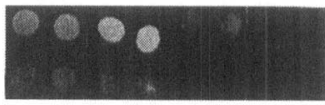

$\operatorname{pdr} 5 \Delta$

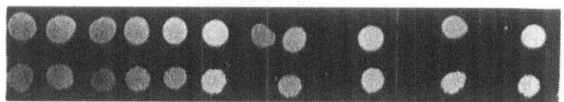

Fig. 3. Pdr5p-mediated emetine resistance in wt, erg $4 \Delta$, erg $3 \Delta$, erg $2 \Delta$, erg $6 \Delta$ and $p d r 5 \Delta$ strains of $S$. cerevisiae. The experiment was essentially done as described in Methods and in the legend to Fig. 2. V, control plasmid; P, PDR5-overexpressing plasmid.

$21 \mathrm{~min}$. At this time point, both erg $2 \Delta$ and $\operatorname{erg} 6 \Delta$ cells were found to be able to efflux rhodamine in the presence of glucose. Very little release of rhodamine was seen at $21 \mathrm{~min}$ in the $\operatorname{erg} 2 \Delta$ and $\operatorname{erg} 6 \Delta$ mutants in the absence of glucose indicating that efflux was not due to mere leakage of the dye across the membranes. The cells lacking Pdr5p ( $p d r 5 \Delta$ strain) showed very low levels of active efflux of the dye even $21 \mathrm{~min}$ after glucose addition (Table 2). This experiment demonstrated that although Pdr5p was functional in these strains, the altered sterol environment had a significant effect on its efflux capabilities.

\section{Does Pdr5p function in erg strains correlate with membrane fluidity?}

Alteration in sterol content of the membrane is known to primarily affect its fluidity. To determine whether the differences in Pdr5p efficiency that we observed in different strains was correlated with membrane fluidity, we measured the membrane fluidity of the strains. This was done with whole cells using both DPH and TMADPH as probes. The fluorescence anisotropies of these probes in membranes correlate inversely with membrane fluidity. Although we did observe an increase in membrane fluidity in erg mutants relative to the wt, we could not find any correlations between the membrane fluidity of these strains and the efficiency of the Pdr5p pump (Table 3).

\section{Oestradiol}

( $\mu \mathrm{g} \mathrm{ml}^{-1}$ ):

Plasmid:

$\frac{75}{V \quad P} \frac{150}{V \quad P} \frac{225}{V \quad P}$

wt

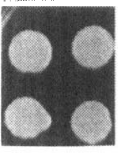

$\operatorname{erg} 4 \Delta$

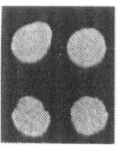

$\operatorname{erg} 3 \Delta$

$\operatorname{erg} 2 \Delta$

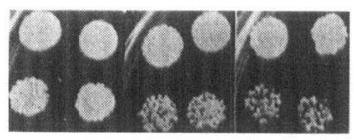

$\operatorname{erg} 6 \Delta$

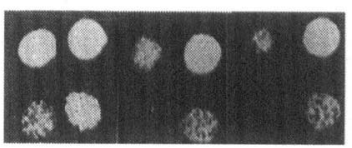

$\operatorname{pdr} 5 \Delta$

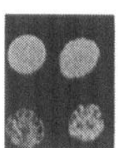

Fig. 4. $\mathrm{Pdr} 5 \mathrm{p}$-mediated $\beta$-oestradiol resistance in $w t$, erg $4 \Delta$, $\operatorname{erg} 3 \Delta$, erg $2 \Delta$, erg $6 \Delta$ and pdr5 $\Delta$ strains of $S$. cerevisiae. The experiment was essentially done as described in Methods and in the legend to Fig. 2. V, control plasmid; P, PDR5-overexpressing plasmid.

\section{Pdr5p pump efficiency in a phosphatidylserine- deficient mutant}

As alteration of the sterol composition led to a significant reduction in the ability of $\mathrm{Pdr} 5 \mathrm{p}$ to confer drug resistance, we wished to determine if alteration in the phospholipid composition of the membrane might also affect Pdr5p efficiency. We therefore constructed a cho1 $\Delta$ strain in the same background. These cells are choline auxotrophs but are otherwise viable. CHO1 encodes phosphatidylserine synthase and its disruption results in the membrane being completely devoid of phosphatidylserine (Hikiji et al., 1988). The lack of phosphatidylserine is largely compensated for by an increase in the phosphatidylinositol and phosphatidylcholine levels (Atkinson et al., 1980).

When we overexpressed Pdr5p from a multicopy plasmid in such a cho1s strain in a similar manner to that done for erg mutants, we observed that the plasmid bearing PDR5 was highly unstable, making a comparison of the drug resistance profiles of Pdr5p difficult to study (data not shown). We therefore carried out the 
Table 2. Pdr5p-mediated energy-dependent efflux of rhodamine $6 \mathrm{G}$ in $\mathrm{wt}$, erg $4 \Delta$, erg $3 \Delta, \operatorname{erg} 2 \Delta$, erg $6 \Delta$ and pdr $5 \Delta$ strains of $S$. cerevisiae

Cells were harvested, prepared and loaded with rhodamine 6G as described in Methods. Rhodamine $6 \mathrm{G}$ efflux was initated by adding $1 \mathrm{mM}$ glucose. The experiment was repeated twice and the values given are of a representative experiment.

\begin{tabular}{|c|c|c|c|}
\hline \multirow[t]{2}{*}{ Strains } & \multicolumn{3}{|c|}{$\begin{array}{l}\text { Rhodamine 6G fluorescence intensity } \\
\text { at }(\min ):\end{array}$} \\
\hline & 0 & 7 & 21 \\
\hline wt - glucose & 112 & 143 & 170 \\
\hline$w t+$ glucose & 123 & 473 & 795 \\
\hline $\operatorname{erg} 4 \Delta-$ glucose & 114 & 133 & 143 \\
\hline $\operatorname{erg} 4 \Delta+$ glucose & 116 & 245 & 226 \\
\hline $\operatorname{erg} 3 \Delta-$ glucose & 119 & 133 & 147 \\
\hline $\operatorname{erg} 3 \Delta+$ glucose & 134 & 358 & 608 \\
\hline $\operatorname{erg} 2 \Delta-$ glucose & 121 & 151 & 172 \\
\hline $\operatorname{erg} 2 \Delta+$ glucose & 107 & 158 & 352 \\
\hline erg6 $6 \Delta-$ glucose & 120 & 142 & 154 \\
\hline $\operatorname{erg} 6 \Delta+g l$ ucose & 120 & 170 & 290 \\
\hline$p d r 5 \Delta$-glucose & 120 & 152 & 174 \\
\hline$p d r 5 \Delta+$ glucose & 114 & 147 & 200 \\
\hline
\end{tabular}

Table 3. Fluorescence anisotropies of DPH and TMA$\mathrm{DPH}$ in $\mathrm{wt}$, erg $4 \Delta, \operatorname{erg} 3 \Delta, \operatorname{erg} 2 \Delta$ and erg $6 \Delta$ strains of $S$. cerevisiae

Measurements were carried out on whole cells and fluorescence anisotropy values were calculated as described in Methods. The values represent the mean of three experiments \pm SD (each experiment was done in triplicate).

\begin{tabular}{|c|c|c|}
\hline Strain & $\mathrm{DPH}$ & TMA-DPH \\
\hline wt & $0.388 \pm 0.056$ & $0.561 \pm 0.055$ \\
\hline $\operatorname{erg} 4 \Delta$ & $0.346 \pm 0.032$ & $0.559 \pm 0.042$ \\
\hline $\operatorname{erg} 3 \Delta$ & $0 \cdot 291 \pm 0.009$ & $0.490 \pm 0.020$ \\
\hline $\operatorname{erg} 2 \Delta$ & $0 \cdot 300 \pm 0 \cdot 012$ & $0.505 \pm 0.030$ \\
\hline $\operatorname{erg} 6 \Delta$ & $0 \cdot 344 \pm 0 \cdot 020$ & $0.513 \pm 0.024$ \\
\hline
\end{tabular}

analysis in a strain that is not deficient in the vacuolar proteases PEP4 and PRB1. In this background, the plasmid was stable, which allowed the comparison of Pdr5p overexpression in this strain (Fig. 5).

The $c h o 1 \Delta$ strain was mildly resistant to cycloheximide as compared to the wt strain, as seen in Fig. 5. Pdr $5 \mathrm{p}$ overexpression in the cho1 $\Delta$ strain was, though, able to confer resistance to cycloheximide to a significant level; however, it did not reach the wt levels of resistance.

In the case of crystal violet, the cho1s strain was significantly more resistant as compared to the wt cells,
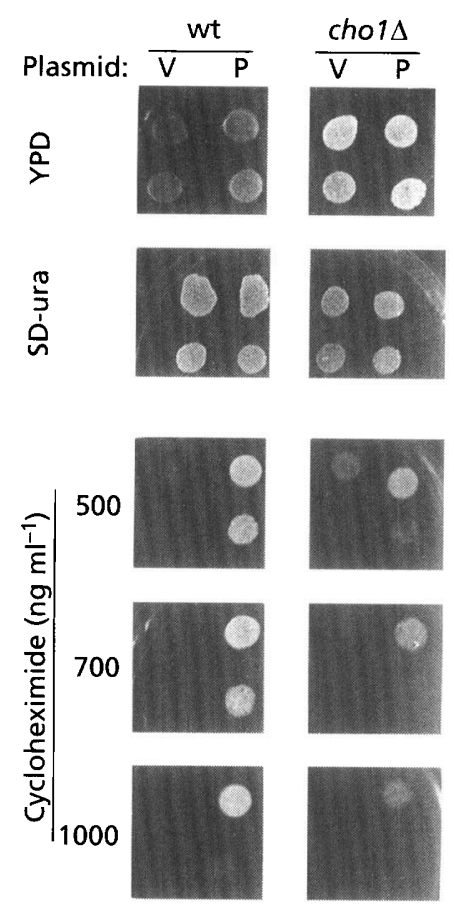

Fig. 5. Stability of PDR5-overexpressing plasmid in wt (ABC154) and cho1 $\triangle(A B C 625)$ strains of $S$. cerevisiae. wt and cho1 strains were transformed with either the control plasmid YEplac195 (V) or the PDR5-overexpressing plasmid PDR5/YEplac195 (P). The wt transformants and the cho1 transformants were grown to exponential phase in SD-ura broth and SD-ura broth supplemented with $1 \mathrm{mM}$ choline, repsectively. Cells were spotted onto plates of YPD, SD-ura containing $1 \mathrm{mM}$ choline, and YPD containing different concentrations of cycloheximide as described in the legend to Fig. 2. The growth pattern of the first two suspensions $\left(10^{7}\right.$ and $10^{6}$ celis $\mathrm{ml}^{-1}$ ) is shown in the first and second rows, respectively.

and the level of resistance conferred by Pdr $5 \mathrm{p}$ was also significantly higher than that in the wt strain (Fig. 6). This drug selectivity indicates that lipid composition also plays a role in modulating the level of drug resistance conferred by the Pdr5p pump, and in a drugspecific manner.

\section{DISCUSSION}

In this report, we have investigated whether the pleiotropic drug sensitivity of erg mutants might in part result from an altered functioning of the yeast MDR pump, $\operatorname{Pdr} 5 \mathrm{p}$. These erg strains are used extensively in different drug-based studies (Graham et al., 1993; Hemenway \& Heitman, 1996) and the tacit assumption has been that these strains are hyperpermeable to different drugs. However, the studies described in this report indicate that this assumption is not entirely valid and one also has to take into account the fact that the efflux pumps have a reduced efficiency in these backgrounds. We have not examined the localization of Pdr5p in these strains and it is possible that the reduced efficiency of $\operatorname{Pdr} 5 p$ in 


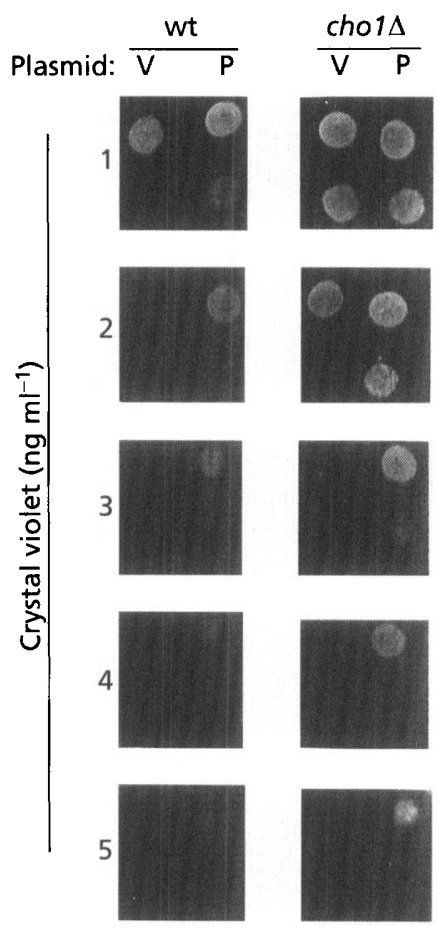

Fig. 6. Pdr5p-mediated crystal violet resistance in wt and cho1 $1 \Delta$ (ABC 625) strains of $S$. cerevisiae. The experiment was essentially done as described in Methods and in the legend to Fig. 5. V, control plasmid; P, PDR5-overexpressing plasmid.

altered sterol backgrounds could be due to partial mislocalization of the protein. However, complete mislocalization had not ocurred since the pumps could mediate efflux of some of the drugs, although to lesser extents. Some other important conclusions may be drawn from this study. Firstly, the drug resistance profiles were affected in a manner that was dependent on both the strain background and the drug being tested. Thus the erg6 $6 \Delta$ strain could efflux some, but not all, of the drugs. This suggests that although the lipid bilayer is not the primary determinant for substrate specificity, it plays an important role in modulating this specificity in addition to modulating efficiency. Furthermore, previous studies have indicated that the ability of the MDR pump to mediate efflux of a drug depends on the permeability of the membrane to the drug (Eytan et al., 1996, 1997), but we observed that the reduced ability of Pdr5p to efflux drugs in different strains is not dependent solely on the permeability of the membrane towards the drugs.

Our results also highlight the importance of evaluating the function and substrate specificity of MDR pumps over a range of drug concentrations to attain more complete resistance profiles.

The decreased efflux of drugs in the erg $6 \Delta$ strain leading to the drug hypersensitivity phenotype is in contrast to the cation-sensitivity phenotype of this strain, where the $\mathrm{Li}^{+}$and $\mathrm{Na}^{+}$hypersensitivity was primarily due to increased uptake rather than the decreased efflux (Welihinda et al., 1994).erg $6 \Delta$ cells also exhibit defective tryptophan uptake, presumably due to a defect in tryptophan permease (Gaber et al., 1989). In addition to this, the transport kinetics of a few other permeases of yeast is also known to be affected by the lipid composition of the membrane (Keenan et al., 1982; Calderbank et al., 1985).

Recently, Egner et al. (1998) have initated detailed studies of mutants of Pdr5p with altered function by random mutagenesis. As mutants with altered substrate specificity were observed to occur in no single domains, and a few of the mutants were also found to have defects in folding, these authors have suggested a possible role for the folded structure of $\operatorname{Pdr} 5 \mathrm{p}$ as a major determinant of its diverse substrate specificity. Lipids have been shown to function as molecular chaperones (Bogdanov et al., 1996) and it is possible that in strains with altered lipid/ergosterol content, some defect in folding of membrane proteins may occur and that this might be partly responsible for both their altered efficiency and their altered substrate specificity.

The observation that $\operatorname{Pdr} 5 \mathrm{p}$ overexpression in a phosphatidylserine-deficient mutant in a proteasedeficient background led to significant differences in plasmid stability suggests that overexpression of this protein is, for some reason, deleterious to cells in this background. Mammalian P-gps have also been shown to destabilize membranes (Arsenault et al., 1988) and it may be interesting to examine whether the destabilization is affected by the composition of the membrane itself.

The present study, for the first time, reports that the reduced efficiency of the MDR pump, $\operatorname{Pdr} 5 \mathrm{p}$, in $S$. cerevisiae erg strains is responsible to a significant extent for their pleiotropic drug-sensitive phenotypes. This observation is of great significance in view of the fact that the majority of antifungal compounds that are being extensively used to treat fungal infections inhibit the enzymes of the ergosterol biosynthetic pathway. Thus it will be very interesting to study whether the reduced efficiency of the MDR pumps of these yeasts might allow use of other drugs in combating the fungal infection. The present study, if extended to pathogenic yeast-like Candida spp., may shed some light on the complex drug resistance phenomenon of this yeast, and will stimulate further dissection of the role of the membrane in the functioning of MDR pumps.

\section{ACKNOWLEDGEMENTS}

We are indebted to Dr K. Kuchler for providing us with the PDR5 plasmid and disruptants. We also thank Drs C. Marcireau, L. Parks, J. Heinisch, H. Pelham, S. Henry and E. Jones for their generous gifts of strains and plasmids. We would like to thank Mr G. Subba Rao, Mr Bharat L. Dixit and other members of Dr C. M. Gupta's laboratory for helping us in conducting rhodamine efflux studies and the membrane fluidity studies. R. Kaur is a Senior Research Fellow of the Council of Scientific and Industrial Research, India. This 
work was supported in part by a Grant-in-aid project (BT/R\&D/15/40/93) from the Department of Biotechnology, Government of India.

\section{REFERENCES}

Arsenault, A. L., Ling, V. \& Kartner, N. (1988). Altered plasma membrane ultrastructure in multidrug-resistant cells. Biochim Biophys Acta 938, 315-321.

Atkinson, K. D., Jensen, B., Kolat, A. I., Storm, E. M., Henry, S. A. \& Fogel, S. (1980). Yeast mutants auxotrophic for choline or ethanolamine. J Bacteriol 141, 558-564.

Balzi, E., Wang, M., Leterme, S., Van Dyck, L. \& Goffeau, A. (1994). $P D R 5$, a novel yeast multidrug resistance conferring transporter controlled by the transcription regulator PDR1. J Biol Chem 269, 2206-2214.

Bard, M., Lees, N. D., Burrows, L. S. \& Kleinhans, F. W. (1978). Differences in crystal violet uptake and cation-induced death among yeast sterol mutants. J Bacteriol 135, 1146-1148.

Baudin, A., Kalogeropoulos, O. O., Denouei, A., Lacroute, F. \& Cullin, C. (1993). A simple and efficient method for direct gene deletion in Saccharomyces cerevisiae. Nucleic Acids Res 21, 3329-3330

Beaudet, L. \& Gros, P. (1995). Functional 'dissection of Pglycoprotein nucleotide-binding domains in chimeric and mutant proteins. J Biol Chem 270, 17159-17170.

Bissinger, P. H. \& Kuchler, K. (1994). Molecular cloning and expression of the Saccharomyces cerevisiae STS1 gene product. $J$ Biol Chem 269, 4180-4186.

Bogdanov, M., Sun, J., Kaback, H. R. \& Dowhan, W. (1996). A phospholipid acts as a chaperone in assembly of a membrane transport protein. J Biol Chem 271, 11615-11618.

Borst, P. \& Schinkel, A. H. (1997). Genetic dissection of the function of mammalian P-glycoproteins. Trends Genet 13, 217-222.

Calderbank, J., Keenan, M. H. J. \& Rose, A. H. (1985). Plasmamembrane phospholipid unsaturation affects expression of the general amino-acid permease in Saccharomyces cerevisiae Y185. J Gen Microbiol 131, 57-65.

Doige, C. A., Yu, X. \& Sharom, F. J. (1993). The effects of lipids and detergents on ATPase-active P-glycoprotein. Biochim Biophys Acta 1146, 65-72.

Egner, R. \& Kuchler, K. (1996). The yeast multidrug transporter Pdr5 of the plasma membrane is ubiquitinated prior to endocytosis and degradation in the vacuole. FEBS Lett 378, 177-181.

Egner, R., Mahe, Y., Pandjaitan, R. \& Kuchler, K. (1995). Endocytosis and vacuolar degradation of plasma membranelocalized Pdr.5-ATP binding cassette multidrug transporter in Saccharomyces cerevisiae. Mol Cell Biol 15, 5879-5887.

Egner, R., Rosenthal, F. E., Kralli, A., Sanglard, D. \& Kuchler, K. (1998). Genetic separation of FK 506 susceptibility and drug transport in the yeast Pdr5 ATP-binding cassette multidrug resistance transporter. Mol Biol Cell 9, 523-543.

Eytan, G. D., Regev, R., Oren, G. \& Assaraf, Y. G. (1996). The role of passive transbilayer drug movement in multidrug resistance and its modulation. J Biol Chem 271, 12897-12902.

Eytan, G. D., Regev, R., Oren, G., Hurwitz, C. D. \& Assaraf, Y. G. (1997). Efficiency of P-glycoprotein-mediated exclusion of rhodamine dyes from multidrug-resistant cells is determined by their passive transmembrane movement rate. Eur J Biochem 248, 104-112.

Gaber, R. F., Copple, D. M., Kennedy, B. K., Vidal, M. \& Bard, M.
(1989). The yeast gene ERG6 is required for normal membrane function but is not essential for biosynthesis of the cell-cyclesparking sterol. Mol Cell Biol 9, 3447-3456.

Gietz, R. D. \& Sugino, A. (1988). New yeast-Escherichia coli shuttle vectors constructed with in vitro mutagenized yeast genes lacking six-base pair restriction sites. Gene 74, 527-534.

Gottesman, M. M. \& Pastan, I. (1993). Biochemistry of multidrug resistance mediated by the multidrug transporter. Annu Rev Biochem 62, 385-427.

Gottesman, M. M., Pastan, I. \& Ambudkar, A. (1996). P-glycoprotein and multidrug resistance. Curr Opin Genet Dev 6, $610-617$.

Graham, T. R., Scott, P. A. \& Emr, S. D. (1993). Brefeldin A reversibly blocks early but not late protein transport steps in the yeast secretory pathway. EMBO J 12, 869-877.

Haggerty, D. F., Kalra, V. K., Popjak, G., Reynolds, E. E. \& Chiappelli, F. (1978). Fluorescence-polarization measurements on normal and mutant human skin fibroblasts. Arch Biochem Biophys 189, 51-62.

Hanna, M., Brault, M., Kwan, T., Kast, C. \& Gros, P. (1996). Mutagenesis of transmembrane domain 11 of P-glycoprotein by alanine scanning. Biochemistry 35, 3625-3635.

Hemenway, C. S. \& Heitman, J. (1996). Immunosuppressant target protein FKBP12 is required for P-glycoprotein function in yeast. J Biol Chem 271, 18527-18534.

Hikiji, T., Miura, K., Kiyono, K., Shibuya, I. \& Ohta, A. (1988). Disruption of the CHO1 gene encoding phosphatidylserine synthase in Saccharomyces cerevisiae. J Biochem 104, 894-900.

Hirata, D., Yano, K., Miyahara, K. \& Miyakawa, T. (1994). Saccharomyces cerevisiae YDR1, which encodes a member of the ATP-binding cassette (ABC) superfamily, is required for multidrug resistance. Curr Genet 26, 285-294.

Ito, H., Fukuda, Y., Murata, K. \& Kimura, A. (1983). Transformation of intact yeast cells treated with alkali cations. $J$ Bacteriol 153, 163-168.

Keenan, M. H. J., Rose, A. H. \& Silverman, B. W. (1982). Effect of plasma-membrane phospholipid unsaturation on solute transport into Saccharomyces cerevisiae NCYC 366. J Gen Microbiol 128, 2547-2556.

Kolaczkowski, M., Rest, M. V., Kolaczkowska, A. C., Soumillion, J. P., Konings, W. N. \& Goffeau, A. (1996). Anticancer drugs, ionophoric peptides, and steroids as substrates of the yeast multidrug transporter Pdr5p. J Biol Chem 271, 31543-31548.

Kralli, A., Bohen, S. P. \& Yamamoto, K. R. (1995). LEM1, an ATPbinding-cassette transporter selectively modulates the biological potency of steroid horomones. Proc Natl Acad Sci USA 92, $4701-4705$.

Kuchler, K. \& Thorner, J. (1992). Functional expression of human MDR1 in the yeast Saccharomyces cerevisiae. Proc Natl Acad Sci USA 89, 2302-2306.

Kuhry, J. G., Duportial, G., Bronner, C. \& Lautriat, G. (1985). Plasma membrene fluidity measurements on whole living cells by fluorescence anisotropy of trimethylammonium diphenylhexatriene. Biochim Biophys Acta 845, 60-67.

Kwan, T. \& Gros, P. (1998). Mutational analysis of the Pglycoprotein first intracellular loop and flanking transmembrane domains. Biochemistry 37, 3337-3350.

Lees, N. D., Skaggs, B., Kirsch, D. R. \& Bard, M. (1995). Cloning of the late genes in the ergosterol biosynthetic pathway of Saccharomyces cerevisiae - a review. Lipids 30, 221-226.

Loo, T. W. \& Clarke, D. M. (1993). Functional consequences of 
proline mutations in the predicted transmembrane domain of $\mathrm{P}$. glycoprotein. J Biol Chem 268, 3143-3149.

Mahe, Y., Lemoine, Y. \& Kuchler, K. (1996). The ATP binding cassette transporters Pdr5 and Snq2 of Saccharomyces cerevisiae can mediate transport of steroids in vivo. J Biol Chem 271, 25167-25172.

Obrenovitch, A., Sene, C., Negre, M. T. \& Monsigny, M. (1978). Fluorescence polarization of 1,6-diphenyl-1,3,5-hexatriene embedded in membranes of mouse leukemic L 1210 cells during the cell cycle. FEBS Lett 88, 187-191.

Rose, M. D., Winston, F. \& Hieter, P. (1990). Methods in Yeast Genetics: a Laboratory Course Manual. Cold Spring Harbor, NY: Cold Spring Harbor Laboratory.

Saeki, T., Shimabuku, A. M., Azuma, Y., Shibano, Y., Komano, T. \& Ueda, K. (1991). Expression of human P-glycoprotein in yeast cells - effects of membrane component sterols on the activity of $\mathrm{P}$ glycoprotein. Agric Biol Chem 55, 1859-1865.

Saeki, T., Shimabuku, A. M., Ueda, K. \& Komano, T. (1992).
Specific drug binding by purified lipid-reconstituted P-glycoprotein: dependence on the lipid composition. Biochim Biophys Acta 1107, 105-110.

Sambrook, J., Fritsch, E. F. \& Maniatis, T. (1989). Molecular Cloning: a Laboratory Manual, 2nd edn. Cold Spring Harbor, NY: Cold Spring Harbor Laboratory.

Sharom, F. J. (1997). The P-glycoprotein efflux pump: how does it transport drugs? J Membr Biol 160, 161-175.

Urbatsch, I. L. \& Senior, A. E. (1995). Effects of lipids on ATPase activity of purified chinese hamster $\mathbf{P}$ glycoprotein. Arch Biochem Biophys 316, 135-140.

Welihinda, A. A., Beavis, A. D. \& Trumbly, R. J. (1994). Mutations in LIS1 (ERG6) gene confer increased sodium and lithium uptake in Saccharomyces cerevisiae. Biochim Biophys Acta 1193, 107-117.

Received 22 September 1998; revised 27 November 1998; accepted 18 December 1998. 